\title{
Suppression of $\mathbf{T}$-cell function in gastric cancer patients after total gastrectomy with splenectomy: implications of splenic autotransplantation
}

\author{
Kiyotaka Okuno, Akira Tanaka, Hironori Shigeoka, Norihiko Hirai, Isao Kawai, Yoshinori Kitano, \\ and MASAYUKi YASUTOMi \\ First Department of Surgery, Kinki University School of Medicine, 377-2 Ohno-higashi, Osaka-sayama, Osaka 589-8511, Japan
}

\begin{abstract}
:
Background. In Japan since the 1960s, patients with gastric cancer have routinely had splenectomy combined with gastrectomy to ensure that lymph node dissection is complete. However, the influence of splenectomy on long-term immunity is unclear.

Methods. Forty-nine gastric cancer patients who underwent total gastrectomy for cure with $(n=25)$ and without $(n=24)$ splenectomy were assessed for immunologic function, including the proportion of lymphocyte subsets, purified protein derivative from tuberculin (PPD) response, natural killer (NK) activity, and phytohemagglutinin (PHA) response.

Results. Peripheral T-cell mediated functions, e.g., PPD and PHA response, were significantly suppressed in patients who underwent gastrectomy with splenectomy compared with those who had gastrectomy alone. Decreased T-cell subsets (CD 3+, 4+, 8+) and increased NK cell subsets (CD 16+, $57+)$ were observed in patients who underwent splenectomy. Patients who did not undergo splenectomy had immunologic responses within the normal range.

Conclusions. Splenectomy decreased T-cell mediated responses over the long term. As a potential means to correct this T-cell dysfunction in patients with splenectomy, splenic autotransplantation should be considered in future research.
\end{abstract}

Key words: splenectomy, gastric cancer, T-cell function, splenic autotransplantation

Offprint requests to: $\mathrm{K}$. Okuno

Received for publication on Dec. 2, 1998; accepted on Feb. 1, 1999

\section{Introduction}

The role of splenectomy in the surgical management of gastric cancer is still controversial. The concept of extended lymph node dissection and its contribution to long-term survival is supported by many institutions, especially those in Japan, Korea, Taiwan, and parts of Europe [1-3]. Splenectomy is often performed to facilitate lymphadenectomy, but the possibility of a negative impact of splenectomy on survival has been raised in several studies [4-6]. The spleen is an important component of the reticuloendothelial system, and it plays a central role in host defenses against infection, by the production of antibodies and opsonins and recruitment of complement $[7,8]$. In addition, the spleen serves as a site of T- and B-cell interaction, which is critical for the secondary immune response to a previously presented antigen [8]. The well established risk of overwhelming infection in asplenic children, and the much smaller but significant risk in adults, is testimony to these important functions [9].

To assess the long-term effect of splenectomy, we investigated peripheral immune function in patients with gastric cancer who underwent splenectomy and gastrectomy.

\section{Patients and methods}

From 1986 to 1995,1012 patients were admitted to the Department of Surgery, Kinki University Hospital, for the treatment of gastric cancer. Of these, 229 patients underwent total gastrectomy for cure; 133 patients underwent splenectomy at the time of total gastrectomy and 96 did not (58\% and 42\%, respectively). Of these patients, 49 had a follow-up of more than 2 years following curative gastrectomy; all agreed to immunologic assessment and were entered into the current study. Twenty five-patients underwent splenectomy at the 
Table 1. Patient characteristics

\begin{tabular}{lccl}
\hline & $\begin{array}{c}\text { Splenectomy } \\
(n=25)\end{array}$ & $\begin{array}{c}\text { No splenectomy } \\
(n=24)\end{array}$ & P \\
\hline Age (years, mean $\pm \mathrm{SD})$ & $58.7 \pm 11.5$ & $59.1 \pm 11.4$ & $\mathrm{NS}$ \\
Sex (M:F) & $14: 11$ & $15: 9$ & $\mathrm{NS}$ \\
Stage I a $+\mathrm{b}$ & 9 & 15 & NS $(P=0.103)$ \\
$\quad$ II & 4 & 5 & \\
$\quad$ IIIa $+\mathrm{b}$ & 10 & 4 & \\
IVa & 2 & 0 & $\mathrm{NS}$ \\
Reconstitution & 16 & 13 & \\
IP & 3 & 6 & \\
DT & 6 & 5 & \\
R-Y & & & \\
\hline
\end{tabular}

IP, Jejunal interposition; DT, double tract; R-Y, Roux-en-Y methods; NS, not significant

time of gastrectomy, and 24 did not (Table 1). Although the splenectomy group showed more advanced stages of disease than the no splenectomy group, the difference was not significant $(P=0.103)$, and there were no significant differences in other variables between the groups.

\section{Surgical technique}

The surgical procedures throughout this period were performed by several attending surgeons and three supervisors (K.O., A.T., and M.Y.). Depending on the location and size of the tumor, total gastrectomy with or without splenectomy was performed.

\section{Isolation of peripheral blood lymphocytes}

Peripheral blood lymphocytes (PBL) were prepared by centrifugation on a sucrose polymer density cushion. Briefly, $20 \mathrm{ml}$ of heparinized venous blood was diluted 1:2 with balanced saline solution and layered onto a lymphocyte separation medium (LSM; Bionetics, Kensington, MD, USA) at 2 vol of diluted blood on $1 \mathrm{vol}$ of LSM. The tubes were centrifuged for $15 \mathrm{~min}$ at $20^{\circ} \mathrm{C}$ with an interface force of $400 \mathrm{~g}$. Cells at the interface were collected and pooled.

\section{Monoclonal antibodies and flow cytometric analysis}

The monoclonal antibodies used for flow cytometry studies included anti-CD3, anti-CD4, anti-CD8, antiCD16, and anti-CD57. All antibodies were purchased from Becton-Dickinson (Mountain View, CA, USA). Cells $\left(10^{6}\right)$ were stained with monoclonal antibody at $4{ }^{\circ} \mathrm{C}$ for $30 \mathrm{~min}$. After extensive washing, the cells were further incubated with fluorescein isothiocyanate (FITC)-conjugated goat antimouse antibody at $4{ }^{\circ} \mathrm{C}$ for $30 \mathrm{~min}$. After being washed, the cells were analyzed on a flow cytometer (FACScan; Becton-Dickinson).
Phytohemagglutinin (PHA) response

Responding lymphocytes $\left(10^{5}\right)$ were incubated in $0.2 \mathrm{ml}$ of conditioned medium in 96-well microtest plates (Iwaki Glass, Osaka, Japan). PHA-P (Difco Laboratories, Detroit, MI, USA) was added at a final concentration of $15 \mathrm{mg} / \mathrm{l}$. After $72 \mathrm{~h}, 0.5 \mu \mathrm{Ci}$ of ${ }^{3} \mathrm{H}$-thymidine was added to each culture and the culture was harvested $24 \mathrm{~h}$ later.

\section{Natural killer activity}

Natural killer (NK) cytotoxicity was determined against K562 targets by a chromium-51 release assay, as previously described [10]. Briefly, various numbers of effector cells were incubated for $4 \mathrm{~h}$ in the presence of targets previously labelled with ${ }^{51} \mathrm{Cr}\left(\mathrm{Na}_{2}{ }^{51} \mathrm{CrO}_{4}, 37 \mathrm{MBq} / \mathrm{ml}\right.$; New England Nuclear, Boston, MA, USA). The culture supernatants were harvested with the Skatron-Titertek system (Skatron AS, Lierbyen, Norway) and counted in a gamma counter. The percent specific cytotoxicity was calculated by the equation: $\%$ cytotoxicity $=100 \times$ (experimental release - spontaneous release) / (maximal release - spontaneous release).

\section{Purified protein derivative from tuberculin (PPD) skin test}

PPD $(0.5 \mathrm{mg} / \mathrm{l}$; Nippon BCG, Tokyo, Japan) was injected $(0.1 \mathrm{ml})$ in the forearm of patients, and the injection sites were measured after $48 \mathrm{~h}$. Skin reactions were measured and expressed as mean diameter (longitudinal length + vertical length).

\section{Statistics}

Data values are presented as means \pm SD. Statistical analyses were performed with a Macintosh LC $630 \mathrm{com}-$ puter and StatView software (Brain Power, Calabasas, 
Table 2. Analysis of surface markers in PBL

\begin{tabular}{clcc}
\hline & Splenectomy & No splenectomy & $t$-test \\
\hline CD 3 & $44.6 \pm 15.9$ & $70.7 \pm 5.8$ & $P<0.05$ \\
4 & $33.8 \pm 8.3$ & $50.8 \pm 5.9$ & $P<0.05$ \\
8 & $28.7 \pm 11.5$ & $24.8 \pm 6.9$ & $\mathrm{NS}$ \\
CD 16 & $29.4 \pm 7.4$ & $18.4 \pm 4.5$ & $P<0.05$ \\
57 & $41.2 \pm 10.3$ & $27.7 \pm 5.5$ & $P<0.05$
\end{tabular}

Peripheral blood lymphocytes (PBL) from gastric cancer patients after curative gastrectomy with/without splenectomy were assessed for cell surface markers by flow cytometry. The percentage of positive cells (mean $\pm \mathrm{SD}$ ) was calculated in each group NS, Not significant

CA, USA) employing the unpaired Student's $t$-test with Welch's correction. The $\chi^{2}$ test with continuity correction was used for the comparison of variables between the splenectomized and non-splenectomized patient groups. A $P$ value of $<0.05$ was considered significant.

\section{Results}

\section{Phenotypic analysis of $P B L$}

The phenotypic patterns of PBL from patients without splenectomy were normal. In contrast, in patients who had undergone splenectomy the percentages of CD3+ and $\mathrm{CD} 4+$ cells were significantly decreased, while CD16+ and CD57+ cells were significantly increased (Table 2).

\section{Flow cytometric analysis of $\mathrm{CD} 16+$ and $\mathrm{CD} 57+$}

Increased CD16+ /or CD57+ PBL in splenectomized patients are shown in Fig. 1. Strongly positive fractions of CD $16+$ and CD57+ (\% positive cells: $54 \%$ and $42 \%$, respectively) were characteristically observed in patients who received splenectomy. These high fractions were not found in PBLs from non-splenectomized cancer patients or healthy controls.

\section{NK activity in $P B L$}

NK activity against K562 target cells was assessed by a standard ${ }^{51} \mathrm{Cr}$-release assay. NK activity in splectomized patients was stronger than that in patients without splenectomy (Fig. 2), a not-surprising result given the phenotype above.

\section{T-cell-dependent responses in vitro and in vivo}

As representative T-cell-dependent responses in vitro and in vivo, PHA-induced proliferation and the PPD skin test, respectively, were assessed in all patients. PHA responses in splenectomized patients were sup- pressed compared with results in patients without splenectomy and controls (Fig. 3, left). In the same fashion, PPD skin test respones in splenectomized patients were also suppressed compared with results in patients without splenectomy or controls (Fig. 3, right).

\section{Discussion}

Adjacent organ resection, including splenectomy, has been done frequently in Japan since the early 1960s to complete lymph node dissection in patients with gastric cancer [1]. This approach was based on the lymphatic flow of the stomach, pancreas, and diaphragm, as reported by lnoue in 1936 [11]. A study of sequential sections of the distal pancreas and splenic hilum showed that remnant nodes were easily detected along the splenic artery. Kawaguchi et al. [12] reviewed 90 patients with total gastrectomy with pancreaticosplenectomy for cure and reported that $24.4 \%$ of the patients had one or more diseased nodes at the splenic hilum, a greater percentage of positive nodes than along the left gastric artery.

In a review of the Japanese experience with splenectomy by Okajima and lsozaki [13], the incidence of hilar nodal involvement was $15.5 \%$ for upper-third cancers, and $20.7 \%$ when the entire stomach was involved. These rates are influenced by the extent of the primary cancer. Thus, the location and stage of the tumor have a major effect on the need for splenectomy to ensure adequate hilar lymph node extirpation.

The concept of an effective, elective splenectomy is governed by numerous associated tumor factors. In a review by Stipa et al. [14] of 646 patients undergoing curative gastrectomy, splenectomy was associated with significantly impaired survival by univariate analysis. In a multivariate analysis of clinical, pathologic, and treatment-related variables, the only independent predictors of death were cirrhosis, distant lymph node involvement, and total gastrectomy; significance was lost for splenectomy and lymphadenectomy. Wanebo et al. [15] summarized the surgical management of gastric cancer in more than 700 hospitals across the United States. The data indicated that $21.1 \%$ of all reported patients with gastrectomy for stomach cancer included splenectomy. The observed 5-year survival rate for patients who underwent splenectomy was $20.9 \%$ and for patients without splenectomy it was $31 \%$. Survival rates were significantly impaired by splenectomy in patients with stage II or III, but not for patients with stage I or IV disease. The pattern of recurrence in patients with splenectomy was somewhat different from that in patients without splenectomy, with a greater number of patients developing distant metastases, $29 \%$ vs $15.5 \%$, respectively. In the current study, the 5-year survival rate 


\section{CD16}

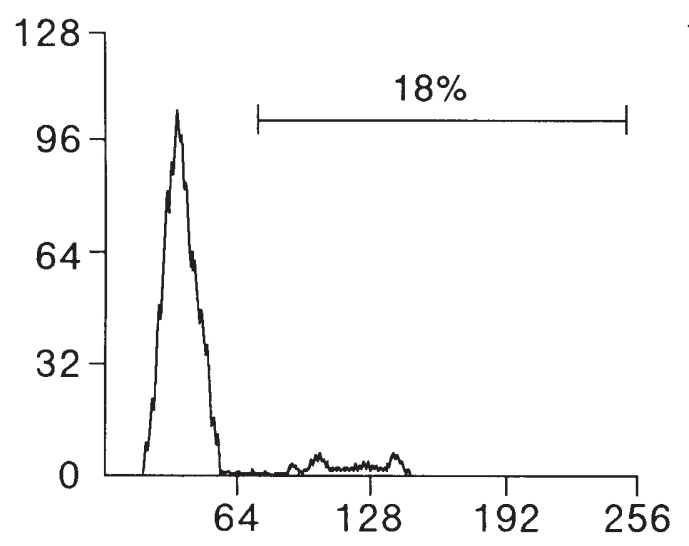

CD57

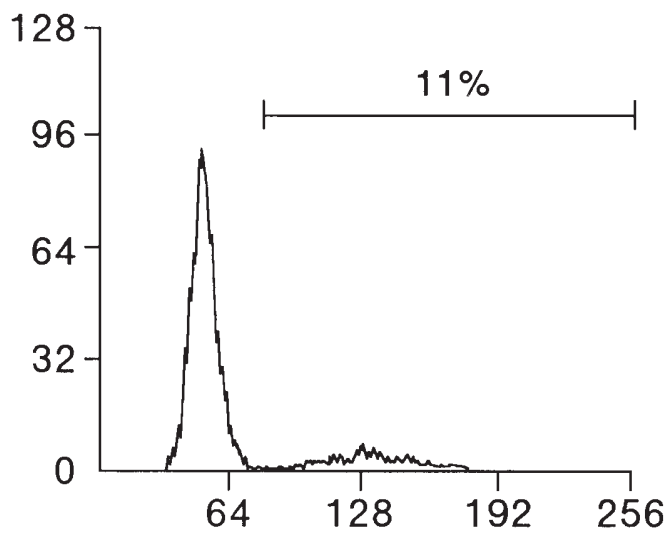

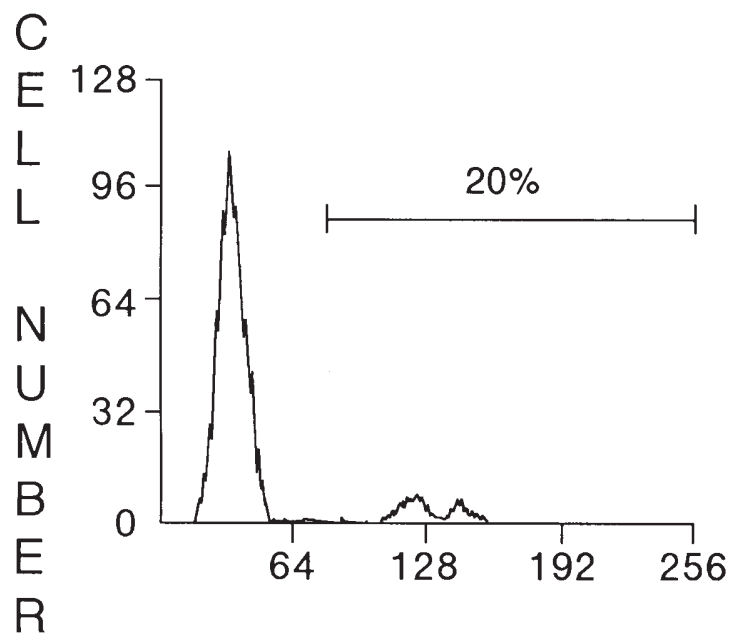
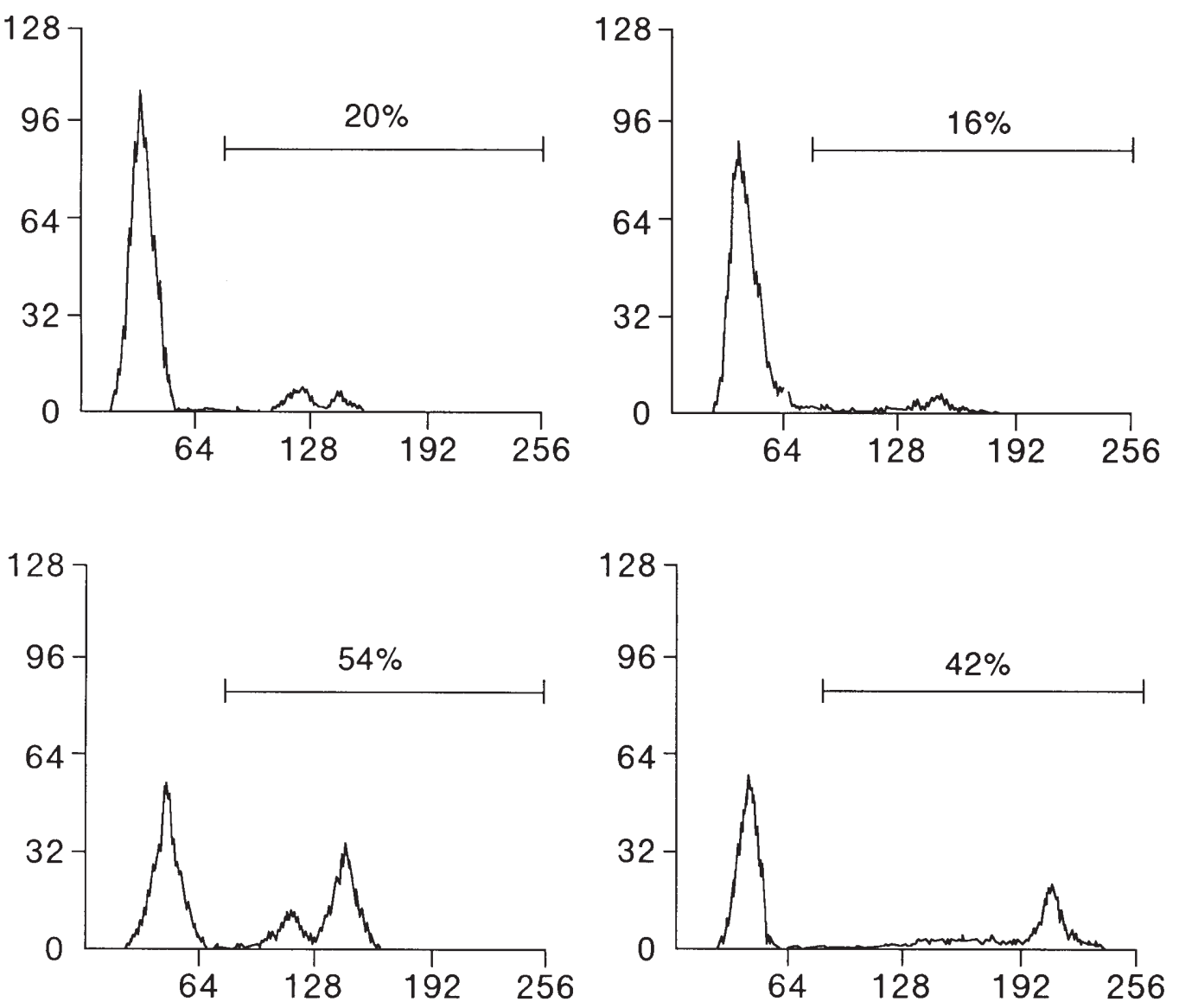

FLUORESCENCE INTENSITY

Fig. 1. Representative flow cytometric pattern of CD16+, and CD57+ peripheral blood lymphocytes (PBL) from patients after gastrectomy with/without splenectomy. PBL from gastric cancer patients with splenectomy (lower), without splenectomy (middle), and from healthy controls (upper) were stained with fluorescein isothiocyanate (FITC) conjugated anti-CD16 or anti-CD57 antibodies. The percentages of positive cells are shown 
of the patients who underwent splenectomy was less than that of the patients who did not undergo splenectomy (50.8\% vs $62.9 \%$, respectively). However, this is a smallscale retrospective study, and the patients who underwent splenectomy necessarily had a more advanced tumor stage than the patients without splenectomy (stages III and IV, 12/25 and 4/24, respectively). Therefore, we cannot draw conclusions about the impact of splenectomy on survival in this study.

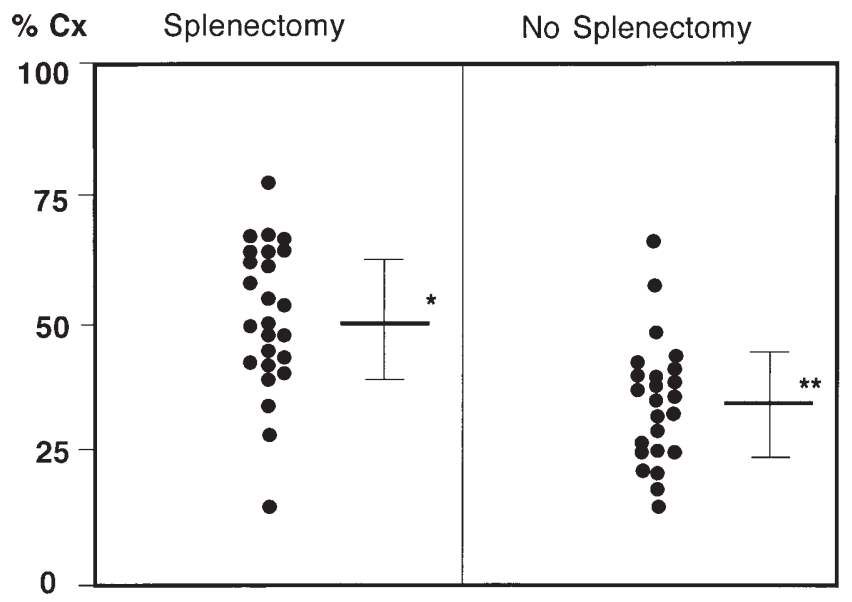

Fig. 2. Natural killer $(N K)$ activity in PBL from patients with (left) and without splenectomy (right). Each dot denotes NK activity (\% lysis [\% $C x$ ] at an effector-to-target ratio of 40 ) for an individual patient. Bars represent means $\pm \mathrm{SD} * \mathrm{vs} * * P<$ 0.01

\section{PHA-induced proliferation}

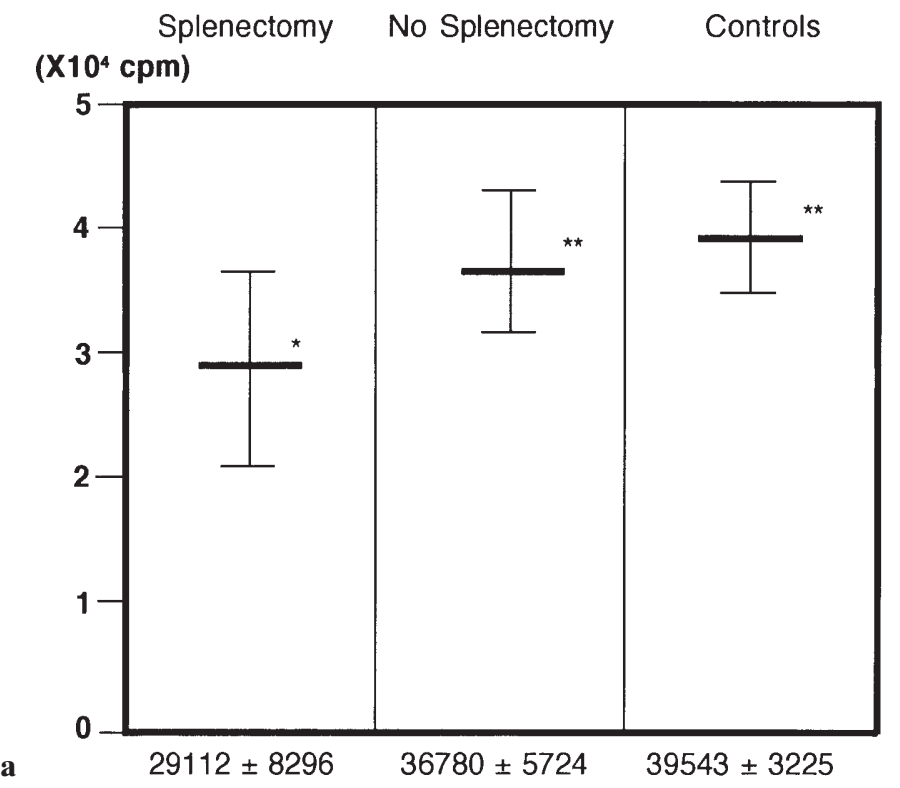

The mechanisms of the possible long-term effects of splenectomy on survival in cancer patients are not known. However, splenectomized patients have diminished antibody responses to particulate antigens, are deficient in tuftsin and phagocytosis-promoting peptide, and have decreased levels of lgM properdin. In the current study, we have shown that T-cell function in splenectomized patients is impaired, and NK activity is augmented long-term. Overall, PBL counts were preserved in patients who underwent splenectomy. However, the proportion of CD3 + cells and the Tcell-dependent responses were decreased. In contrast, CD16+ or CD57+ cells and NK activity were increased. The intensity of CD16+ or CD57+ labeling by flow cytometry was high; this fraction is not usually detected in the PBL of healthy persons or in gastric cancer patients who have not undergone splenectomy (Fig. 2). It is conceivable that this highly mature fraction of lymphocytes which possess NK activity is usually captured and cleared by the spleen. If we could assess the preoperative immune response of patients, we could ascertain the immune response more precisely. At present, we cannot conclude that these changes worsen survival in splenectomized patients. However, longterm T-cell suppression in splenectomized patients is unlikely to be beneficial for immune surveillance.

In order to resolve this dilemma of achieving, complete lymph node removal in the splenic hilum without loss of splenic function, we are pursuing the technique of splenic autotransplantation. Splenic autotran-

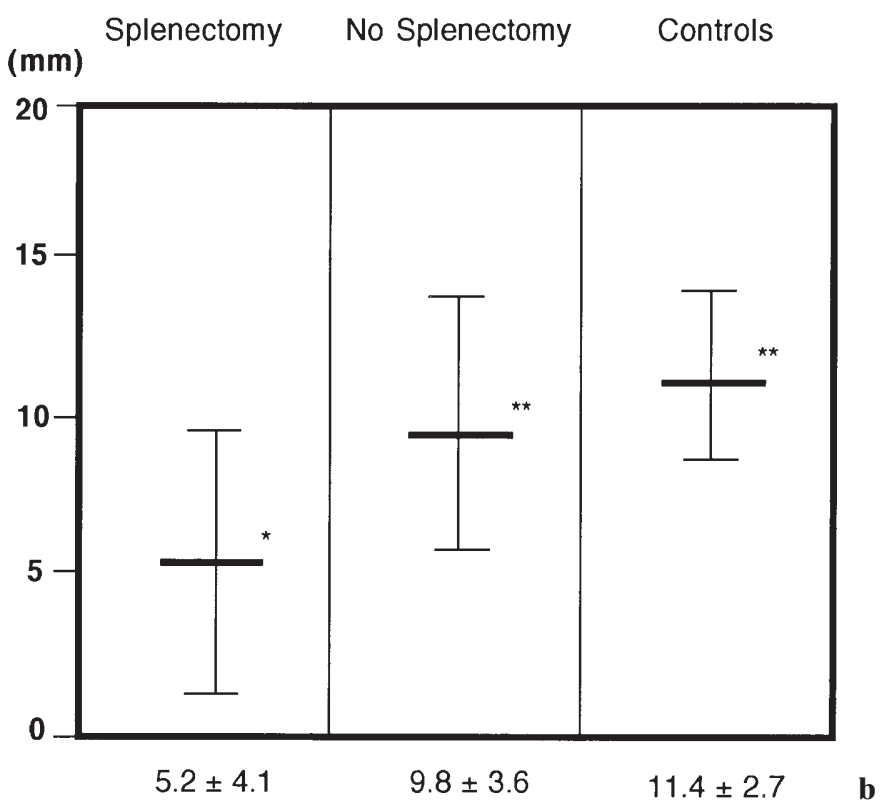

Fig. 3. T-cell-dependent responses in vitro and in vivo. Controls Age-matched healthy controls $(n=7)$. Bars represent means \pm $\mathrm{SD}$ in each group. $P H A$, phytohemagglutinin; $P P D$, purified protein derivative from tuberculin, $*$ vs $* * P<0.05$ 
splantation was originally advocated for patients with severe trauma who would otherwise require splenectomy [16]. For this subset of patients, splenic salvage by autotransplantation theoretically preserves the critical role of the spleen in the host's defenses against infection. We have begun splenic autotransplantation in patients with gastric cancer who undergo total gastrectomy with splenectomy using an isolated segment of the small intestine, according to the methods of Shokouh-Amiri et al. [17]. This procedure preserves intact circulation and produces a higher index of regeneration than other previously reported sites. In fact, we have observed evidence of splenic function with normalization of postsplenectomy thrombocytosis, peripheral lymphocyte subsets, and peripheral blood smears in patients following splenic autotransplantation (Okuno et al., manuscript in preparation).

We believe that complete lymphadenectomy including splenectomy, followed by splenic autotransplantation, will improve survival and the quality of life in patients with advanced gastric cancer who undergo potentially curative resection.

Acknowledgments. This work was supported in part by Grants-in-Aid for Scientific Research (C) from the Ministry of Education, Science and Culture (08671495) of Japan.

\section{References}

1. Maruyama K, Okabayashi K, Kinoshita T. Progress in gastric cancer surgery and its limits of radicality. World J Surg 1987;11:418-25.

2. Kim J, Kwoni J, Oh ST, Ynag HK. Result s of surgery on 6539 gastric cancer patients and immunochemo surgery as the best treatment of advanced gastric cancer. Ann Surg 1992;216:26979.

3. Roder JD, Böttcher K, Siewert JR, Busch R, Hermanek P, Meyer $\mathrm{HJ}$, et al. Prognostic factors in gastric carcinoma: results of German gastric carcinoma study 1992. Cancer 1993;72:2089-97.

4. Sugimachi K, Kodama Y, Kumashiro R, Kanematsu T, Noda S, Inokuchi K. Critical evaluation of prophylactic splenectomy in a total gastrectomy for gastric carcinoma. Gann 1980;71:704-9.

5. Bonenkamp JJ, Songun J, Hermans J, Sasako M, Welvaart K, Plukker JT, et al. Randomized comparison of morbidity after D1 and D2 dissection for gastric cancer in 996 Dutch patients. Lancet 1995;345:745-8.

6. Yoshino K, Haruyama K, Nakamura S, Matsumoto S, Yamada Y, Isobe $\mathrm{K}$, et al. Evaluation of splenectomy for gastric carcinoma. Jpn J Gastroenterol Surg 1979;12:944-9.

7. Rose WF. The spleen as filter. N Engl J Med 1987;317:705-6.

8. Brown EJ, Hosea SW, Frank MM. The role of complement in the localization of pneumococci in the splenic reticuloendothelial system during experimental bacteremia. J Immunol 1981;16:2230-5.

9. Llende M, Santiago-Delpin EA, Lavergne J. Immunobiological consequences of splenectomy: a review. J Surg Res 1986;40:85-94.

10. Okuno K, Takagi H, Nakamura T, Nakamura Y, Iwasa Z, Yasutomi M. Treatment for unresectable hepatoma via selective hepatic arterial infusion of lymphokine-activated killer cells generated from autologous spleen cells. Cancer 1986;58:1001-6.

11. Inoue Y. Lymphatics of the stomach, duodenum and diaphragm (in Japanese). Acta Anat Nippon 1936;9:35-120.

12. Kawaguchi M, Muto K, Nashimoto A, Miyashita K, Tanaka O, Sasaki K. Clinical implication of splenectomy associated with the operation for gastric cancer (in Japanese). Rinsho Geka. J Clin Surg 1983;38:185-8.

13. Okajima K, Isozaki H. Splenectomy for treatment of gastric cancer: Japanese experience. World J Surg 1995;19:537-40.

14. Stipa S, DiGiorgio A, Ferri M, Botti C. Results of curative gastrectomy for carcinoma. J Am Coll Surg 1994;17:567-72.

15. Wanebo HJ, Kennedy BJ, Winchester DP, Stewart AK, Fremgen AM. Role of splenectomy in gastric cancer surgery: adverse effect of elective splenectomy on longterm survival. J Am Coll Surg 1997;185:177-84.

16. Pisters PW, Pachter HL. Autologous splenic transplantation for splenic trauma. Ann Surg 1994;219:225-35.

17. Shokouh-Amiri MH, Bayat M, Rahimi-Saber S, Lindkaer Jensen S, Kerndrup G. Autotransplantation of splenic tissue in an isolated segment of small intestine. Br J Surg 1992;79:1327-9. 